\title{
Using participatory design methods with ubiquitous computing technologies
}

\author{
Jim Wood \\ Goldsmiths, University of London \\ Lewisham Way, London \\ jim.wood@gold.ac.uk
}

\begin{abstract}
This paper outlines one of the projects from my PhD research which proposes the use of tangible design methods in participatory and creative scenarios to enable the conceptualization of uses for "the internet of things" - that is to say the contemporary interest in connecting everyday objects together in networks, for the home, in social settings or over the internet. This is related to the increasing use of RFID (Radio Frequency Identification) tags to label items and the possibilities for peer-to-peer tag communication between devices that is enabled in the Near Field Communication (NFC) standard. It represents one part of a larger research that also includes projects about digitally embodied objects as tools, and commonsbased networks as peer learning environments.
\end{abstract}

Design methods, Participatory design, Ubiquitous interaction, Interactive Prototyping, Internet of Things.

\section{INTRODUCTION}

The original vision of Ubiquitous computing [9] has inspired much research and discourse since the 1990s. However that proposal for "Calm Technology" has sometimes been superseded by an over-interpretation of ubiquitous as meaning disappearing and invisible. Recent revisions have restated the importance of engaging and social experiences, [7] as exemplified by Interaction Design and Tangible Media. [5] The integration of computing into everyday spaces and objects has created ever smaller and more ingenious use of embedded technologies. But where are the everyday methods to bring this new era of $\mathrm{HCl}$ into everyday life and play? How can we enable engagement with ubiquitous computing platforms and networks in a participatory way, such as in play or creative expression?

To explore this proposition I have designed a set of artefacts for a design methods toolkit, 't2t' that will be discussed in this paper. This aims to act as a simple analogue of a system, and to un-pack how to interact with this technology, in its invisibility, by enabling creative participants to engage with it and to create their own scenarios.

\subsection{Design Methods}

The conceptual approach for this project is based on the idea of design methods cards. These are commonly used for teaching or facilitating workshops, brainstorming or role-playing, and would be used as cues or instructions for starting activities or describing the ideation process. IDEO offers a commercially available version [3]. These cards use photographs and short texts to introduce ideas, or starting points of conversations or brainstorming sessions, they are usually categorized around subjects or actions. Whilst IDEO's product is based in their own experience, an evolution of the process in using these cards is to develop this process from your own practice and make your own. With that view I thought it more interesting to leave these as more open ended and adaptable than a "finished" printed set of cards.

The discipline of Participatory Design provides a useful framework for engaging users in designing or co-designing by enacting with props or games that represent scenarios [2]. These types of cards, as discussed are best designed for group activities, or what Johansson \& Linde call "exploratory design games" [5].

\subsection{Notation}

In developing a graphic and symbolic method for describing interactions between technological artefacts inspiration was taken from UML (Unified Modelling Language) [8] the graphical notation system that is used to model software processes, particularly in object oriented programming. In the latest specification UML.2.0 13 diagram types are specified, within these the most relevant to this project would be the behaviour 'use case diagram' and interaction 'sequence diagram'.

Another initial reference was a design study into RFID interactions by Timo Arnall and Schulze \& Webb, [1], designers working with a view towards how technology is mediated through design and communication. This project develops a visual reference language for symbolising the differences between mobile, wireless and touch interactions. 
This leads to the design problem: how can we take a visual code for interaction and make it into a set of modelling methods, which are represented by a diagrammatic language? It is from this point that the proposal for the design methods cards toolkit was developed.

\section{DESIGN SPECIFICATION AND PROTOTYPE}

The specification for a new set of method cards takes as a model the web-based digital service Touchatag and its proposal to enable a service for the Internet of Things [7]. Touchatag provides an internet-based service that enables the connection of physical artefacts to an authentication service, via the use of RFID (Radio Frequency Identification) tagged stickers, or QR codes (QR ref). Thus everyday objects can act as a 'physical hyperlinks' or a trigger to open an application on the PC, send an email etc, authenticating tags through their USB reader.

Though they are joining into the debate about 'The internet of Things' the focus seems to have been more on "the Internet' than the things. From a designer/maker perspective this would seem to be a paradox. So, it is the intention to enable design methods for 'things in the internet'.
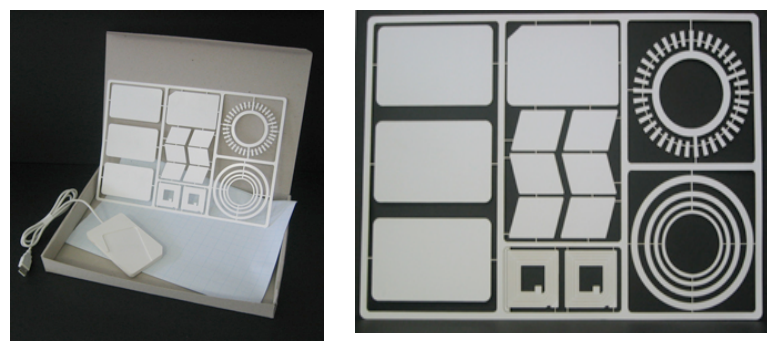

Figure 1\& 2: 't2t' card prototype (the author)

Working from the references above a set of methods cards was designed that were based on a visual language inspired from a mixture of the UML schema and the embodiment of the related objects themselves. This is given the code name "t2t" to refer to the tactile and tacit way of acting with the cards, and also in reference to the $p 2 p$ (Peer-to-peer) mode that is part of the NFC protocol description. The toolkit was prepared as a box of artefacts and instructions that are to be used in participatory workshop settings with a mixed range of users, but mostly expected to be of a non-technical expertise.

A physical template card was made that resembled a toy model kit. This was laser cut from paper and plastic, and designed in a fashion that the set of shapes can be delivered on a frame which they can then be snapped off from. The tangible quality of the cards encourages a playful and experiential focus on the subject matter, that is seen as important to get users to think less about what is happening in the computer, but more what can happen in their own environments. Scenarios were then sketched, and latterly made into animated movie clips that demonstrated example uses.

\section{FINDINGS}

The toolkit has been tested in limited 'in-house settings', and it its being prepared for use in workshops in summer 2010. It is also envisaged that this toolkit will be made available as a template for downloading in a digital image or CAD format. This may allow dissemination to a wide range of participants, and compliments the wider aims of my $\mathrm{PhD}$ research to encourage commonsbased design and making through participatory methods.

\section{REFERENCES}

[1] Arnall, T. (2005) A Graphic Language for Touch,http://www.elasticspace.com/presentati ons/graphic language touch rfid nfc.pdf (retrieved 10.05.2010)

[2] Brandt, E. (2006) Designing exploratory design games: a framework for participation in Participatory Design? In Proceedings of the Ninth Conference on Participatory Design: Expanding Boundaries in Design - Volume 1, Trento, Italy, August 01 - 05, 2006, p 57-66, ACM, New York.

[3] IDEO, (2002) Methods Cards, http://www.ideo.com/work/item/methodcards/ (retrieved 10.05.2010)

[4] Ishii, H. (2004) Bottles: A Transparent Interface as a Tribute to Mark Weiser, IEICE Transactions on Information and Systems, vol. E87-D, no. 6.

[5] Johansson, M. Linde,P. (2005) Playful Collaborative Exploration: New Research Practice in Participatory Design Journal of Research Practice, Vol.1,Issue.1

[6] Rogers, Y. (2006) "Moving on from Weiser's Vision of Calm Computing: Engaging UbiComp Experiences" Ubiquitous Computing: 8th International Conference, UbiComp 2006, Orange County, CA, USA, September 17-21, 2006, Proceedings. 1st ed. Springer

[7] Touchatag, http://www.touchatag.com (retrieved 10.05.2010)

[8] UML website, Object Management Group http://www.uml.org/ (retrieved 10.05.2010)

[9] Weiser, M. (1991) The Computer for the 21st century, Scientific American, vol.265, no.3, p.94-104 